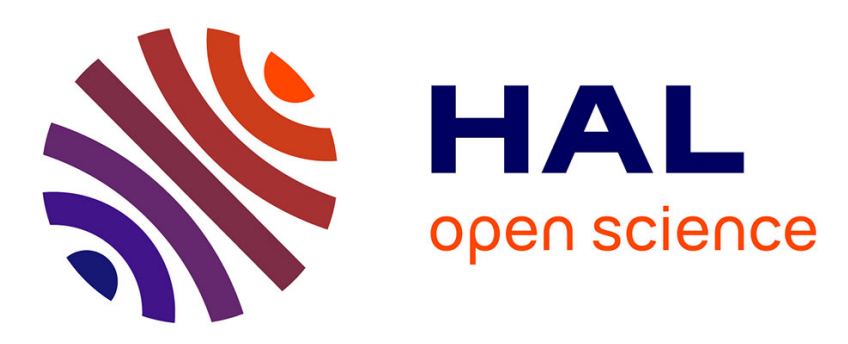

\title{
Eco-concevoir pour l'industrie alimentaire : quelles spécificités ?
}

Gwenola Bertoluci, Gilles Trystram

\section{To cite this version:}

Gwenola Bertoluci, Gilles Trystram. Eco-concevoir pour l'industrie alimentaire : quelles spécificités ?. Marché et Organisations, 2013, Ecoconception, conception et innovation, 17 (1), p 123-135. 10.3917/maorg.017.0123 . hal-01251844

\section{HAL Id: hal-01251844 \\ https://hal.science/hal-01251844}

Submitted on 6 Jan 2016

HAL is a multi-disciplinary open access archive for the deposit and dissemination of scientific research documents, whether they are published or not. The documents may come from teaching and research institutions in France or abroad, or from public or private research centers.
L'archive ouverte pluridisciplinaire HAL, est destinée au dépôt et à la diffusion de documents scientifiques de niveau recherche, publiés ou non, émanant des établissements d'enseignement et de recherche français ou étrangers, des laboratoires publics ou privés. 


\title{
ECO-CONCEVOIR POUR L'INDUSTRIE ALIMENTAIRE : QUELLES SPECIFICITES?
}

\author{
Gwenola BERTOLUCI \\ LGI Centrale Paris \\ gwenola.bertoluci@ecp.fr
}

Gilles TRYSTRAM

UMR 1145, AgroParisTech, INRA, Cnam gilles.trystram@agroparistech.fr

\section{INTRODUCTION}

La fonction principale de l'alimentation est de répondre à un besoin fondamental de l'humanité : fournir des apports caloriques et nutritionnels qui contribuent à l'espérance d'une vie en bonne santé. Dans les pays développés, il est entendu que c'est le rôle du politique d'assurer que la quantité, la qualité et le coût des aliments permettent à leurs populations de satisfaire à ces exigences vitales. La révolution verte, la politique Européenne et l'industrialisation de la transformation alimentaire qui ont suivi la seconde guerre mondiale ont simultanément concouru à rejeter dans l'ombre le spectre des famines et carences alimentaires pour les habitants des pays Européens les plus développés. D’autres aspects ont alors pris le pas dans les attentes des consommateurs des pays développés ; la recherche de sécurité, de plaisir, de surprise, de rêves, de santé.... Parallèlement les pays en croissance découvrent à leur tour ces dimensions et aspirent à en bénéficier, tandis que d'autres restent dans une situation de dépendance telle que seul l'apport nutritif compte. L'ensemble induit une situation de contraste, vois de tension dans un contexte alimentaire mondialisé. Tant de dimensions que l'offre alimentaire doit satisfaire alors que les grands bouleversements liés à la réduction de la disponibilité des ressources (énergétique, aquatique, de la biomasse, ....) qui secouent notre société réinterrogent notre capacité à maintenir nos modes de consommation.

Dans cet article nous examinerons dans un premier temps, plus en profondeur, les spécificités de l'offre alimentaire qui impliquent, selon nous, que l'éco-conception de ces produits recouvre des dimensions similaires à celles d'autres produits manufacturés mais également des aspects tout à fait spécifiques. Nous construirons ainsi une proposition de définition de l'éco-conception de ces produits.

Dans une seconde partie, nous esquisserons une synthèse des approches conduites dans les filières agroalimentaires, par le passé et actuellement, qui contribuent à cette éco-conception, que cette propriété ait été ou non initialement le but de la conduite de ces actions. Pour ce faire nous balayerons les différentes grandes étapes du cycle de vie des produits alimentaires et les actions conduites par les acteurs principaux de ces différentes étapes.

\section{Les spécificités de l'éco-conception alimentaire}

Dans sa thèse, S. Le Pochat ((Le Pochat 2005)) déclarait «Dans les pays riches, une société de subsistance, liée à la satisfaction des besoins vitaux, a été remplacée par une société de consommation, rendue accessible grâce à la révolution 
industrielle». Le cas de l'offre alimentaire des pays riches de l'Europe illustre parfaitement cette citation. La diversification et l'enrichissement effrénés de cette offre démontrent de la nécessité pour les producteurs d'une politique de différenciation due à une saturation de ces marchés qui n'offrent plus de croissance. Cette dynamique est doublement pilotée. D'une part par l'amont avec une réduction de la diversité des matières premières utilisées, au bénéfice d'une régularité et d'une productivité accentuées. Cette dynamique est contestée aujourd'hui tant par la demande consommateurs que par la réalité du marché des matières premières. La seconde dynamique est celle de la création de niches de produits, à valeur ajoutée augmentée qui induit que l'outil industriel fabrique de la diversité. L'observatoire de la qualité des Aliments (Ministère de l'agriculture 2012)) produit de nombreuses études qui illustrent cette situation. Les produits résultant sont en général des variations d'un produit de référence.

Il semblait donc que ces marchés ne pouvaient plus être l'enjeu de grands bouleversements. Pourtant la question ancestrale de la sécurité alimentaire a commencé de se reposer officiellement lors des émeutes de la faim de 2007-2008. L'onde de choc immédiate et postérieure à ces événements a mis en lumière l'augmentation du risque de pénurie de ressources (ce que l'on nomme l'insécurité alimentaire) qui menace même nos pays développés dans les décennies à venir. Les origines de ce phénomène sont multiples : croissance de la population mondiale, spéculations boursières ((Economic and Social Development Department 2010)), variations climatologiques, bouleversements géopolitiques, des choix d'usage de terres à des activités autres que nourricières : bâtis, bio produits non alimentaires, restructuration des territoires ... Ce sujet est à traiter au niveau des politiques publiques nationales mais également au niveau de l'offre produit. Les choix d'ingrédients employés notamment les sources de protéines, leur origine géographique et l'efficacité nutritionnelle de ces substances vont devoir être considérés sont l'angle de la durabilité qu'ils induisent.

La création d'une offre alimentaire pour nourrir correctement le plus grand nombre semble donc un objectif initial indiscutable. La prise en compte de nouveaux critères (historiquement sanitaire, sensoriel, nutritionnel) puis aujourd'hui la prise de conscience de l'impact environnemental implique un besoin de définir l'éco-conception alimentaire.

Mais l'offre alimentaire ne se conçoit plus (ou pas encore!) dans nos pays avec pour seul objectif de permettre l'apport calorique et nutritionnel. Elle s'est progressivement complexifiée tandis que la concurrence se développait pour proposer plus de choix, plus de gourmandise, plus de sécurité sanitaire, plus de praticité, autant d'attributs avec lesquels les consommateurs ne sont pas prêts à transiger aujourd'hui. La peur d'une crise alimentaire telle que celle de la vache folle freine l'introduction de nouveaux aliments tout autant que les craintes habituelles de l'homme telles que les défini Claude Fischler vis à vis de la nouveauté alimentaire ((Fischler 1988)). Sur les produits courants les exigences de la traçabilité alimentaire, du nettoyage des installations, des désinfections des matériels, de maintien de la chaine du froid ... sont autant d'obligations qui ont permis de créer une offre saine mais chère en consommations de ressources avec les moyens techniques actuels. Par ailleurs, les limites de la consommation des marchés Bio et Ethiques ne s'imputent pas qu'aux prix de ces produits, le refus de variabilité dans la qualité des produits, ou encore le doute parfois exprimé sur leur qualité gustative, constituent également des freins à leurs achats ((Tagbata and Siriex 2010)). L'éco-conception des produits alimentaires doit s'entendre dans ce cadre indiscutable des perceptions et des peurs des consommateurs et dans celui de plus en plus rigide des textes réglementaires et normatifs qui ont accompagné cette évolution (Codex Alimentarius, International Food Standard, l'Iso 22000, Novel Food...). La sécurité sanitaire constitue un critère d'évaluation de la durabilité dans sa dimension sociale car elle est une demande sociétale incontournable. Elle s'est construite au travers de textes normatifs et par conséquent sur les moyens matériels et informationnels lourds et couteux qu'ils requièrent dans les phases de transformation, stockage et distribution de l'alimentation.

L'éco-conception des produits alimentaires implique de maintenir cette sécurité sanitaire en réduisant les impacts associés aux filières de transformation distribution. 
Ce dernier point nous amène à l'incidence des différentes phases du cycle de vie des produits dans les empreintes environnementales globales qu'ils génèrent. On différencie aisément les profils d'impacts des produits manufacturés selon qu'ils consomment, ou non, des ressources au cours de leurs cycles de vies ((Le Pochat, Bertoluci et al. 2007)). Dans le cas des produits alimentaires, les typologies restent à établir même si des profils peuvent s'envisager notamment en fonction des modes de conservation employés. Ainsi les produits issus des animaux ont toujours un lourd impact énergétique et ceux qui requièrent le maintien d'une chaine du froid sont également prépondérants dans ce domaine. Le tableau 1 illustre cette idée.

\begin{tabular}{|c|c|c|c|c|c|c|c|c|c|}
\hline Produit & Ferme & e $\begin{array}{l}\text { Embal. } \\
\text { primaire }\end{array}$ & $\begin{array}{c}\text { Transp. } \\
\text { De } \\
\text { emballage }\end{array}$ & $\begin{array}{l}\text { Transp. } \\
\text { de } \\
\text { l'usine }\end{array}$ & Vente & $\begin{array}{l}\text { Transp.Mag. } \\
\text { maison }\end{array}$ & $\begin{array}{l}\text { Stockage } \\
\text { maison }\end{array}$ & $\begin{array}{l}\text { Prépar. } \\
\text { maison }\end{array}$ & Total \\
\hline Pain & 11 & 0.4 & 0.6 & 0.4 & 0.2 & 0.4 & 9.0 & 2.0 & 24 \\
\hline Fromage & 5.0 & 0.1 & 0.2 & 0.2 & 0.2 & 0.1 & 0.5 & 0.0 & 6.3 \\
\hline Poisson & 12.0 & 1.1 & 0.4 & 0.3 & 0.4 & 0.1 & 2.0 & 0.7 & 17 \\
\hline $\begin{array}{l}\text { Fruits } \\
\text { Frais }\end{array}$ & 4.0 & 0.6 & 1.1 & 0.7 & 0.4 & 0.4 & 0 & 0 & 7.2 \\
\hline Viande & 65 & 3.4 & 1.5 & 1.7 & 3.0 & 0.5 & 15 & 14 & 104.1 \\
\hline Lait & 13.0 & 3.2 & 1.2 & 3.5 & 2.0 & 0.7 & 14.4 & 0 & 38 \\
\hline Sodas & 3.0 & 4.2 & 1.0 & 0.6 & 0.2 & 0.4 & 2.0 & 0.0 & 11.4 \\
\hline Sucre & 3.0 & 0.3 & 0 & 0.2 & 0.1 & 0.1 & 0 & 0 & 3.7 \\
\hline
\end{tabular}

Tableau 1 : Dépense énergétique d'une semaine de consommation d'un Anglais des aliments recensés (INCPEN 2009).

\section{Les spécificités de l'éco-conception alimentaire}

En conséquence, les impacts environnementaux du champ ou de la ferme sont toujours proportionnellement très conséquents, cependant d'autres dimensions de la vie du produit peuvent être considérées comme sources d'amélioration et notamment les dernières phases du cycle de vie au cours desquelles le comportement du consommateur a une incidence très importante : sur les temps et conditions de stockage notamment au froid, sur la quantité de nourriture jetée à la poubelle, sur le mode de préparation du repas...

Par ailleurs, la création de l'offre alimentaire peut se réexaminer à la lumière de la concomitance de problèmes de malnutrition (excès ou manque d'un ou plusieurs nutriments), qui impactent tant des populations de pays développés que de pays en voies de développement. L'analyse des données historiques montre de quelle manière l'accroissement du niveau vie concours à l'augmentation de la part lipidique dans les régimes alimentaires. Cette évolution conduit à des situations d'accroissement fort de l'obésité dans des pays où la sous nutrition demeure très importante pour une partie de la population ((Combris, Maire et al. 2011)). En effet, la très grande rapidité du changement de régime alimentaire empêche une adaptation progressive des quantités absorbées. De même que la famine tue, la mauvaise adaptation de la diète aux besoins alimentaires réels est une question de santé publique. Hors la composition de cette diète est influencée par le sexe et la catégorie socio Professionnelle des Consommateurs. Ainsi, la diète masculine française a, en moyenne, un impact carbone supérieur à celui des femmes, corrélé à la consommation en viande ((Darmon and Soler 2011)) cependant que cette même consommation est plus faible dans les classes sociales supérieures que dans les classes populaires ((Combris, Maire et al. 2011)). Ce dernier point n'est possible que dans les pays développés comme en France où la part du budget des ménages consacrée à l'alimentation est passée de 21,5\% en 1959 à 13,4 
\% en 2010 alors que ce budget représente 70\% des revenus dans les pays pauvres)((Hanne and Roux 2012)).

Ainsi si de façon générale l'évaluation environnementale des produits montre l'importance du comportement de leur utilisateur sur leur performance environnementale cette réalité est accentuée dans le cas des produits alimentaires.

L'éco-conception des produits alimentaires est indissociable de la conception pour un usage qui limite les pertes liées au stockage, à la préparation, à la mise à la poubelle des ressources alimentaires et de leurs emballages. Ce constat met également en exergue la nécessité de l'évolution du comportement et de la responsabilisation des consommateurs.

La construction du système alimentaire se fait de manière distincte selon les pays. On reconnaît cinq classes différenciantes (Colonna, Fournier et al. 2011)(Tableau 2). Dans la plupart de ces classes, deux filières d'obtention d'aliments se distinguent. D'une part, les filières où l'aliment est simplement conservé ou transformé en une étape (avec une finalité de consommation retardée, le plus souvent). Une seconde filière, plus importante sans doute est celle où la matière première est fractionnée, puis réassemblée pour l'obtention de fonctionnalités diverses. Cette voie d'obtention de l'aliment a donné lieu à la création de la filière, florissante, des ingrédients et additifs au moins partiellement remise en question dans un cadre de durabilité. Deux raisons majeures émergent. D’une part le fait de fractionner atomise la matière première générant potentiellement des déchets ou des coproduits, mettant en œuvre de l'énergie, des solvants potentiellement impactant dans le bilan écologique. D'autre part, parmi les peurs des consommateurs, les inconnues quant aux ingrédients sont importantes. La notion de «Clean label » récemment introduite se positionne dans la mise en œuvre de fonctionnalités à partir de la composition endogène de l'aliment (ou de ses fractions). Cette direction vise alors à remplacer les ingrédients. L'émergence de ce point de vue et de son accompagnement marketing ne doit cependant pas faire négliger une analyse complémentaire. Toute la valorisation de la matière première dans une finalité alimentaire n'est pas possible. Certains coproduits constituants des fractions importantes de l'aliment doivent être valorisés sous forme d'énergie ou employés comme des sources de carbone entrant dans d'autres filières que celles des aliments. La question est alors de savoir si la conception de l'aliment et de sa chaine d'obtention doit se faire uniquement sur l'aliment lui-même ou être considérée dans les valorisations d'autres natures.

L'éco-conception des produits alimentaires est indissociable d'une vision d'ensemble où la valorisation des fractions énergétique et nutritionnelle se combine avec la valorisation des fractions à finalités non alimentaires de types énergie, matériaux, cosmétiques ou chimiques.

\begin{tabular}{|c|l|}
\hline $\begin{array}{c}\text { Nom du système } \\
\text { alimentaire }\end{array}$ & Caractéristiques \\
\hline SA Domestique & $\begin{array}{l}\text { Consommation sur place. } \\
\text { Méconnaissance des pratiques. }\end{array}$ \\
\hline SA Vivrier & $\begin{array}{l}\text { Distances courtes à longues, frein logistique. } \\
\text { Savoir-faire et pratiques, basé sur capacité de } \\
\text { stockage et conservation intrinsèque à la nature } \\
\text { du produit. Peu de transformations ou de } \\
\text { formulation. }\end{array}$ \\
\hline SA Proximité & $\begin{array}{l}\text { Circuit court, peu ou pas de transformation. } \\
\text { Lié aux modes de productions agricoles. } \\
\text { Méconnaissance des pratiques finales. }\end{array}$ \\
\hline SA Qualité & $\begin{array}{l}\text { Bio, éthique, religieux. } \\
\text { Implicite avec usage de labels. } \\
\text { Caractéristiques de niches, formulation souvent } \\
\text { élaborée. }\end{array}$ \\
\hline
\end{tabular}




\begin{tabular}{|c|l|}
\hline $\begin{array}{c}\text { SA Agro } \\
\text { Industriel }\end{array}$ & $\begin{array}{l}\text { Produit de masse, caractérisé par la } \\
\text { consommation retardée Réglementé, image, } \\
\text { forte complexité des formulations }\end{array}$ \\
\hline
\end{tabular}

Tableau 2 : Classement typologique des systèmes alimentaires d'après(Colonna, Fournier et al. 2011)

\section{Des voies explorées pour améliorer l'éco-conception des produits alimentaires}

Depuis la seconde guerre mondiale l'accroissement de la capacité de production alimentaire a été un enjeu majeur des nations. La croissance de la production agricole a été construite sur trois leviers : l'augmentation des rendements (levier essentiel jusqu'à ces dernières années créée par: la mécanisation du travail, l'emploi de produits phytosanitaires et engrais, la sélection des semences, la réduction de la diversité des matières premières), l'augmentation des fréquences de cultures (réduction des temps de jachères) et l'augmentation des surfaces de terres cultivées.

Les taux de croissance des rendements stagnent depuis plusieurs années avec deux causes explicatives: les techniques d'accroissement des rendements employées ces quarante dernières années n'arrivent plus à progresser, plus grave, leur usage a provoqué l'épuisement d'une partie des sols induisant une perte des productions. Les pratiques d'agriculture raisonnée visent à contrecarrer ces effets négatifs, proposant de privilégier un maintien de la production sur le plus long terme au prix de rendements moins élevés des terres cultivées mais aussi en préservant la qualité et la durabilité des sols. Ces pratiques permettent, par ailleurs, de réduire les quantités d'intrants chimiques associés aux cultures et dont les lessivas polluent les nappes phréatiques. Jusqu'à il y a une dizaine d'années ces aspects de gestion de l'agriculture étaient relativement peu pris en compte par les acheteurs des entreprises de transformations alimentaires. Le respect de la réglementation nationale puis Européenne devait être avéré mais la demande allait rarement au-delà. Ces aspects évoluent actuellement avec une nouvelle perception du rôle de l'interaction entre agriculture et qualité du produit final. La notion d'une conception par reverse engineering avec des attentes consommateurs fortes en termes de qualité entendue au sens large (organoleptique, sanitaire, environnementale, sociale...) est de plus en plus ressentie par les entreprises de marques et pour les produits à Marques Distributeurs de qualité supérieure. Cette évolution repose actuellement avec une grande acuité la question des relations d'acteur à acteur au sein des chaines de valeurs qui vont du champ à l'assiette. La remise en cause des natures de relations (déséquilibrées) qui ont concourues à la situation de crise économique actuelle pour une partie des agriculteurs est également indéniablement liée au questionnement sur la sécurité alimentaire des années à venir et du besoin qui en découle pour les marques d'assurer leurs approvisionnements en matières premières.

En parallèle aux changements en cours dans l'amont agricole, l'industrie de la transformation alimentaire ((Proot 2001)) a travaillé au cours des 20 dernières années à la réduction de ses consommations en eau, énergie et sur sa production de déchets. La réduction de consommation en eau a été conduite en modifiant les technologies (par exemple en remplaçant l'eau auparavant employée comme vecteur de transport des chaines de production par des circuits pneumatiques ou gravitaires, par l'utilisation de vapeur d'eau issue des opérations unitaires ultérieures pour le nettoyage des légumes...), par son réusage en cascade, son recyclage, en encore par un travail sur la dynamique des fluides permettant de réduire les pertes et les stagnations dans les tuyaux qui impliquent plus de nettoyages... Des réductions de consommations en énergie ont été atteintes par récupération et ré usage de la chaleur produite (vaporisation de l'eau), par une meilleure maitrise et gestion des phénomènes physiques dans les installations de traitement également. Enfin la valorisation des déchets sous forme de coproduits permet dans certaines filières de réduire non seulement les coûts de gestion des déchets mais également de créer une valeur économique complémentaire. Des propositions ont été construites pour proposer des indicateurs qui agrègent les performances obtenues sur l'eau, l'énergie, et la consommation de ressources 
renouvelables au niveau de l'usine et de la chaine de production afin de les rapporter sur le produit luimême(Gerbens-Leenes, Moll et al. 2003).

Les campagnes visant à réduire les volumes de déchets d'emballage impactent également le secteur alimentaire. Bien que la France peine à rentrer dans les objectifs de la directive européenne sur les emballages (OJEU 1994), de notables progrès ont été accomplis (Figure 1).

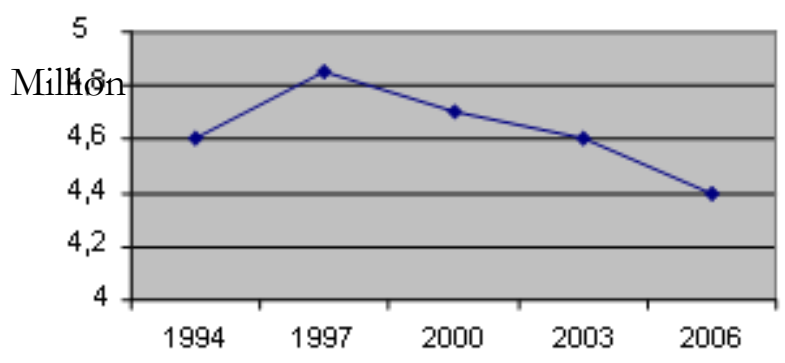

Figure 1: Evolution de la masse d'emballages primaires mis sur le marché Français (Ademe 2007)

L'éco-conception d'emballages passe par la réduction des épaisseurs de matières, par une harmonisation des matériaux plastiques employés si les compatibilités contenant/contenu l'autorisent c'est-à-dire si les migrations possibles entre l'emballage et l'aliment restent conformes aux seuils autorisés par la réglementation alimentaire Européenne. L'usage du plastique recyclé est également autorisé, il peut alors, selon les précautions sanitaires à prendre, être utilisé comme un pourcentage de la masse globale du plastique (applicable que dans le cas du PET - polyéthylène téréphtalate) ou en couche externe d'un emballage multicouches, la face interne demeurant en plastique vierge. Des solutions alternatives de plastiques issus à part entière ou non de matières renouvelables (maïs, canne à sucre, pommes de terre, algues....) ont également été développées. Leur intégration demeure problématique pour différentes raisons dont celle de leur prix beaucoup plus élevé que celui des matériaux issus du pétrole. Ainsi le PLA, principal plastique biosourcé exploité par l'industrie alimentaire, était commercialisé à un prix variant entre 2,3 et 3,4 euros du kilo en 2010, soit le double du prix lui permettant de concurrencer un plastique pétrolier. Par ailleurs, les propriétés de fin de vie de ces matériaux sont variables et complexifient les situations de tris (certains biosourcés polluent les flux de plastiques pétroliers les rendant impropres à une réutilisation) et de gestion des déchets. Certains d'entre eux sont biodégradables, d'autres sont biodispersibles (laissés dans la nature ils s'émiettent et si ils contiennent une part d'origine pétrolière, cette dispersion se révèle extrêmement polluante). Enfin, la biodégradabilité implique la mise en condition particulière d'humidité et de chaleur de l'emballage pour qu'elle puisse s'effectuer dans un délai restreint de quelques semaines. En conséquence un emballage biodégradable abandonné dans la nature constituera une réelle pollution visuelle, problématique pour la marque qu'il porte.

La conception de l'aliment elle-même a évolué au cours des dernières années pour proposer de nouvelles gammes de produits portant la mention de clean label. Le clean label est à la transformation alimentaire ce que l'agriculture raisonnée est à l'agriculture industrialisée. Il s'agit de concevoir une offre qui puisse répondre aux attentes d'une population qui souhaite de "meilleurs » produits sans avoir les moyens et ou l'envie d'aborder les produits biologiques. La notion de Clean Labels recouvre deux dimensions. La première est celle de la promesse d'une communication sincère sur la composition réelle du produit en des termes compréhensibles par les consommateurs. La seconde concerne la composition même des produits dans lesquels les entreprises s'efforcent de réduire ou supprimer les adjonctions de conservateurs et d'exhausteurs de goûts mis en cause dans certains problèmes de santé et globalement considérés comme non "naturels » et donc signant la dimension industrielle des produits proposés par les industries agroalimentaires. Le contre coup de cette évolution des recettes est une augmentation de leur coût de revient par les frais direct (amélioration de la qualité des ingrédients employés pour permettre la suppression des exhausteurs de goûts) et indirects (les coûts de R\&D) afférents. Un des 
enjeux des entreprises engagées dans ces évolutions est de ne pas être amenées à réduire la durée de vie possible de leurs produits sous peine d'accroitre les pertes en distribution et l'insatisfaction des consommateurs au domicile. La maitrise des atmosphères contrôlées dans les emballages, en permettant de ralentir les phénomènes de migrations et de maturation des produits, apportent dans certains cas des solutions à cette problématique (le gaz de l'atmosphère contrôlée n'étant pas considéré par la législation comme un ingrédient ni un additif, il n'empêche pas l'appellation Clean Label).

La question de l'évaluation environnementale des produits alimentaires est un sujet que les entreprises ont actuellement beaucoup de mal à traiter. Ce constat peut s'expliquer par un certain retard mis par le secteur industriel à aborder ce sujet mais également à des spécificités du domaine. Le retard se démontre dans le fait que des sites Européens de production de grandes marques alimentaires peuvent ne pas être encore Iso 14001 ou Emas, situation que l'on ne rencontrera pas dans l'automobile par exemple (Renault a débuté la certification ISO 14001 de ses sites en 1995 et 100\% de ses sites sont aujourd'hui ISO 14001). Or la certification site ((Bertoluci and Le Pochat 2006)) est un premier pas dans la trajectoire d'intégration des préoccupations environnementales dans la culture des entreprises. Au-delà de ce décalage, le manque de données sur les bilans des flux associés aux différentes phases du cycle de vie des produits alimentaires rend difficile toute évaluation environnementale globale. Les données manquantes portent en premier lieu sur les impacts des produits non transformés (végétaux ou animaux) en fonction de leurs conditions d'élevage ou de culture (exemple : le bilan effet de serre d'une tomate cultivée en serre en France pourra être largement supérieure à celui d'une tomate Marocaine cultivée en plein champ et transportée par bateau... mais il faudra connaitre les distances de transport en camion pour différencier réellement les impacts respectifs). Les industries manufacturières travaillent beaucoup sur commandes ouvertes après audits des fournisseurs. Dans l'alimentaire, seules les entreprises travaillant en établissant des contrats avec les agriculteurs sont dans une situation similaire mais en générale elles compétent cet approvisionnement par des achats ponctuels sur le marché. Du fait de l'arrivée de produits de base pouvant porter des empreintes environnementales très variables (culture en plein champs ou pas, durées et modes de transport, traitements phytosanitaires appliqués...) une même entreprise peut proposer des produits finis avec de larges spectres de profils environnementaux d'une période à une autre. Les impacts des procédés de transformations sont eux aussi également mal connus aujourd'hui or les procédés sont multiples et le plus souvent différentes voies sont possibles pour arriver à un produit globalement similaire. Ainsi, pour éliminer les micro-organismes d'un lait employé par la suite dans la fabrication d'un yaourt il sera possible de procéder par un traitement thermique classique, par ultra filtration, par traitement à haute pression... toutes ces voies technologiques fournissent la fonctionnalité attendue, avec des propriétés organoleptiques différentes et des impacts environnementaux variables. Actuellement, l'analyse des impacts de choix de ces moyens de réalisation des opérations unitaires sur l'empreinte environnementale des produits alimentaires, est inconnue.

Ce dernier paragraphe nous amène sur le sujet de l'information environnementale portée par les produits. Déjà présente dans différents pays (Japon, Suède, Angleterre...) elle y est proposée avec des choix d'indicateurs et des méthodes de calculs variables. En France, la plateforme d'étiquetage environnementale Ademe Afnor des produits de grande consommation travaille à la définition d'un étiquetage environnementale en synergie avec les industriels et leurs représentations corporelles. Le groupe a décidé d'expérimenter un étiquetage devant comporter des informations multicritères sur l'eau, l'effet de serre, et l'atteinte à la biodiversité. Ce choix est donc différent de celui l'Angleterre qui a choisi d'étiqueter uniquement l'effet de serre. Une synthèse officielle de l'expérimentation conduite entre 2011 et 2012 est encore attendue. Cependant certains des acteurs privés qui ont pris part à cette expérimentation mettent en avant que deux aspects essentiels doivent évoluer pour qu'un tel étiquetage ait du sens. D'une part, que soient véritablement identifiées les attentes informationnelles des consommateurs en termes de natures, de formes (valeurs absolue ou relative, à savoir par exemple : quantité de carbone émise pour la mise à disposition de ce produit ou part qu'il représente vis-à-vis de l'émission moyenne journalière d'un français) et de support d'informations (emballage, ticket de caisse, 
téléphone portable par lecture d'une puce....) préférés. Ces attentes sont à établir par familles de produits car elles ne sont probablement pas les mêmes pour toutes. Par ailleurs, l'évaluation proposée par la plateforme s'appuie sur des données environnementales seulement alors que sur ces types de produits d'autres questions semblent intéresser une partie des consommateurs, on citera le bien-être animal, la provenance nationale ou régionale des produits et par contre coup les bénéfices de création de valeur en France associés aux produits. Ce chantier de l'étiquetage environnemental parait donc n'en être qu'à ses débuts, mettant essentiellement aujourd'hui en évidence l'insuffisance des connaissances disponibles pour permettre son aboutissement immédiat mais ayant le mérite d'initier la démarche.

\section{Conclusion}

En synthèse, l'éco-conception des produits alimentaires se présente de façon très particulière car bien que la réflexion sur la dimension environnementale se soit engagée plus tardivement que pour les produits manufacturés, elle inclut immédiatement des aspects sociaux (questionnement sur l'intérêt santé pour les consommateurs, le bien-être animal, la reconnaissance des différents acteurs des filières) et économiques (avec l'émergence des circuits courts, des systèmes alternatifs de distribution, le commerce éthique, l'accès aux produits aux plus grand nombre). En conséquence, l'utilisation d'une méthode telle que l'ACV, qui fait aujourd'hui relativement consensus dans les autres secteurs industriels, pour conduire l'évaluation des produits existants ne répond que de façon très partielle aux questionnements qui se posent. Cette situation est renforcée par le manque de données dont disposent les experts pour conduire ces évaluations environnementales. Les travaux en cours devraient conduire à compléter les bases et probablement à structurer des méthodes plus spécifiques d'évaluation dédiées à ces produits et à ce que l'on souhaite savoir de leurs performances environnementales. Cependant notre analyse nous conduit à penser que cette voie ne saurait suffire et que tant les besoins d'informations que l'ampleur des objectifs à atteindre nécessitent une réelle évolution de la création de valeur dans les filières alimentaires et du partenariat entre les acteurs. Les systèmes alimentaires structurés en étapes de fractionnement et d'assemblage génèrent une large variété de flux à destination alimentaire et autres. La gestion intelligente en terme de durabilité de ces coproduits impliquent des approches plus organisationnelles que produits. Le produit ne sera jamais qu'une résultante d'une organisation globale et c'est au niveau des progrès qui peuvent être accomplis sur cette structure générale, sur la manière dont elle gère ses flux de matières et d'informations que de réelles avancées environnementales et sociales peuvent être accomplis.

\section{Bibliographie}

ADEME, 2007,Le gisement des emballages ménagers en France. Angers, Ed. Ademe - Eco EmballageAdelphe, pp35.

BERTOLUCI, G., Le Pochat, S., 2006,Gérer le déploiement des connaissances environnementales pour permettre l'intégration de l'éco-conception dans l'industrie Ingénierie de la conception et cycle de vie des produits. Hermes Publications, Ed. Roucoules, L., Yannou, B., Eynard, B. 
COLONNA, P., FOURNIER, S., TOUZARD, J., 2011, Systèmes alimentaires.Dualine, Durabilité de l'alimentation face à de nouveaux enjeux. Questions de recherche. Ed. C. Esnouf, M. Russel and N. Bricas, INRACIRAD, pp.

COMBRIS P., MAIRE, B., REQUILLART, V., 2011, Consommation et consommateurs.Dualine, Durabilité de l'alimentation face à de nouveaux enjeux. Questions de recherche.Ed.C. Esnouf, M. Russel and N. Bricas, INRA-CIRAD, pp 60-88.

DARMON, N., SOLER, LG., 2011,Impact carbone et qualité nutritionnelle des régimes aliementaires en France Dualine, Durabilité de l'alimentation face à de nowveaux enjeux. Questions de recherche.Ed. C. Esnouf, M. Russel and N. Bricas, INRA-CIRAD, pp 45-58.

ECONOMIC AND SOCIAL DEVELOPMENT DPT, 2010, Price Volatility in Agricultural Markets, FAO Policies Briefs - Economic and Social Perspectives. Retrieved October 2012, from http://www.fao.org/docrep/013/am053e/am053e00.pdf.

FISCHLER, C. (1988). "Food, self and identity." Social Science Information,Vol.27, pp937-953.

GERBENS-LEENES, P.W.,MOLL, H.C., SCHOOT UITERKAMP, A.J.M., 2003, Design and development of a measuring method for environmental sustainability in food production system, Ecological economics, Vol.46, pp 234-248.

HANNE, H., ROUX, N., 2012,Évolution des dépenses et des prix d'alimentation dans la consommation des ménages en France et en Europe depuis 1959, journal DGCRF Eco, DGCRF, pp16.

INCPEN, 2009, Table for one, the energy cost to feed one person. Report, Industry Council for Packaging and the environment, pp27.

LE POCHAT, S., 2005,Intégration de l'éco-conception dans les PME : Proposition d'une méthode d'appropriation de savoir-faire pour la conception environnementale des produits. MAPIE. Chambery, Arts\&Métiers ParisTech. PhD, pp279.

LE POCHAT, S., BERTOLUCI, G., FROELICH, D., 2007, Integrating ecodesign by conducting the change in SMEs, Journal of Cleaner Production Vol.15(7), pp. 671-680.

MINISTERE DE L'AGRICULTURE, 2012, Oqali - Offre et caractéristiques des aliments, http://www.oqali.fr/oqali/, consultation oct. 2012.

OJEU,1994, Packaging and packaging waste, Directive 94/62/EC., Brussel, European Parliament and the Council.

PROOT, J.,2001, Les technologies propres appliquées aux industries agroalimentaires, Rapport ARIST, pp26. 
TAGBATA, D. SIRIEX, L., 2010,L'équitable, le bio et le goût. Quels sont les effets de la double labellisation bio-équitable sur le consentement à payer de consommateurs?

Cabiers Agricultures, Vol.19,pp34-40. 